\title{
Abaloparatide for risk reduction of nonvertebral and vertebral fractures in postmenopausal women with osteoporosis: a network meta-analysis
}

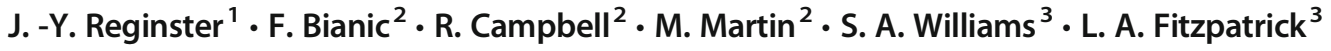

Received: 20 November 2018 / Accepted: 18 March 2019 / Published online: 6 April 2019

(C) The Author(s) 2019

\begin{abstract}
Summary This network meta-analysis assessed the efficacy of abaloparatide versus other treatment options to reduce the risk of fractures in women with postmenopausal osteoporosis. The analysis indicates that abaloparatide reduces the risk of fractures in women with postmenopausal osteoporosis versus placebo and compared with other treatment options. Introduction This network meta-analysis (NMA) assessed the relative efficacy of abaloparatide versus other treatments to reduce the risk of fractures in women with postmenopausal osteoporosis (PMO).

Methods PubMed®, Embase $\AA$, and the Cochrane Central Register of Controlled Trials were searched for randomized controlled trials published before December 20, 2017, that included women with PMO who were eligible to receive interventions for primary or secondary fracture prevention. The NMA was conducted by fracture site (vertebral [VF], nonvertebral [NVF], and wrist), with the relative risk (RR) of fracture versus placebo the main clinical endpoint. The NMA used fixed-effects and randomeffects approaches.

Results A total of 4978 articles were screened, of which 22 were included in the analysis. Compared with other treatments, abaloparatide demonstrated the greatest treatment effect relative to placebo in the VF network $(\mathrm{RR}=0.13$; 95\% credible interval [CrI] 0.04-0.34), the NVF network $(\mathrm{RR}=0.50 ; 95 \% \mathrm{CrI} 0.28-0.85)$, and the wrist fracture network $(\mathrm{RR}=0.39$; CrI $0.15-0.90)$. Treatment ranking showed that abaloparatide had the highest estimated probability of preventing fractures in each of the networks (79\% for VF, $70 \%$ for NVF, and 53\% for wrist fracture) compared with other treatments. Individual networks demonstrated a good level of agreement with direct trial evidence and direct pairwise comparisons.

Conclusions This NMA indicates that abaloparatide reduces the RR of VF, NVF, and wrist fracture in women with PMO with or without prior fracture versus placebo, compared with other treatment options. Limitations include that adverse events and drug costs were not considered, and that generalizability is limited to the trial populations and endpoints included in the NMA.
\end{abstract}

Keywords Abaloparatide $\cdot$ ACTIVE $\cdot$ Fracture prevention $\cdot$ Network meta-analysis $\cdot$ Postmenopausal osteoporosis $\cdot$ Teriparatide

Electronic supplementary material The online version of this article (https://doi.org/10.1007/s00198-019-04947-2) contains supplementary material, which is available to authorized users.

J. -Y. Reginster

jyreginster@ulg.ac.be

Université de Liège, Place du 20 Août 7, 4000 Liège, Belgium

2 Syneos Health, London, UK

3 Radius Health, Inc., Waltham, MA, USA

\section{Introduction}

Osteoporosis is among the most consequential health crises for industrialized countries with aging populations. In 2000, osteoporosis was the cause of more than 8.9 million fractures [1]; of these fragility fractures, 1.6 million involved the hip; 1.7 million, the forearm; and 1.4 million, the vertebrae [1]. In the absence of meaningful preventive measures, approximately one half of Americans aged $>50$ years will be at high risk of osteoporosis by 2020 [2]. Direct costs from osteoporotic fractures in men and women are projected to exceed $\$ 25$ billion by 2025 in the USA, and the cumulative cost of incident fractures 
is expected to reach $\$ 228$ billion for the 10 -year period between 2016 and 2025 [3]. In 2010, the estimated direct costs of osteoporotic fractures in the five largest EU countries (France, Germany, Italy, Spain, and UK) amounted to $€ 29$ billion, and $€ 38.7$ billion in the $27 \mathrm{EU}$ countries [4].

In view of these realities, it is imperative to identify therapeutic agents that can effectively prevent fragility fractures due to osteoporosis, especially in postmenopausal women. The roster of established treatments includes antiresorptive agents, anabolic agents, and agents that have both anabolic and antiresorptive properties. Abaloparatide is an anabolic agent that selectively activates the parathyroid hormone 1 (PTH1)receptor signaling pathway and stimulates bone formation [5]. Abaloparatide is a treatment for postmenopausal women with osteoporosis who are at high risk for fracture or who have failed, or are intolerant to, other available osteoporosis therapies [6]. In the 18-month Abaloparatide Comparator Trial In Vertebral Endpoints (ACTIVE; NCT01343004), women with postmenopausal osteoporosis (PMO) who were randomized to receive daily subcutaneous injection of abaloparatide $(80 \mu \mathrm{g}$; $n=824)$, placebo $(n=821)$, or open-label teriparatide $(20 \mu \mathrm{g}$; $n=818$ ) experienced significant reductions in the risk for new vertebral fractures (VF) and for nonvertebral fractures (NVF): Risk reduction for VF was $86 \%(p<0.001)$, and risk reduction for NVF was $43 \%$ ( $p=0.049)$ [7]. A prespecified exploratory analysis revealed that abaloparatide was also associated with a significantly reduced risk for major osteoporotic fractures versus placebo $(70 \% ; p<0.001)$ and teriparatide $(55 \% ; p=0.03)$ [7]. Risk reduction persisted in these anatomic sites in the extension study of ACTIVE (ACTIVExtend; NCT01657162) after patients on abaloparatide or placebo were switched to alendronate for 24 months [8].

Given the array of agents available for preventing osteoporotic fractures, analysis of their relative safety and efficacy can benefit clinicians looking to individualize treatment for patients. This prompted us to conduct a network meta-analysis (NMA) to identify which osteoporosis treatments exhibit optimal efficacy in postmenopausal women at high risk for fragility fracture. Network meta-analyses employ systematic literature reviews (SLRs) to compare multiple treatments directly within randomized controlled trials (RCTs) and indirectly across trials using a common comparator. Regulatory authorities require that new osteoporosis treatments demonstrate their effect on VFs and NVFs, so we focused our analysis on these events [9].

\section{Methods}

We undertook an SLR to identify all relevant RCTs involving abaloparatide and all pertinent comparators. The main clinical endpoint was the relative risk (RR) of abaloparatide versus placebo and other available treatments to reduce fracture risk. Treatment ranking according to performance for each outcome was the secondary endpoint. The global ranking matrix was populated by the proportion of simulations in which each treatment is ranked best (i.e., the treatment is associated with the smaller risk of fractures), second best, third, and so on.

\section{Study selection}

Three electronic databases-PubMed ${ }^{\circledR}$, Embase $®$, and the Cochrane Central Register of Controlled Trials (CENTRAL) - were searched for RCTs published prior to December 20, 2017. Studies were selected that met predefined eligibility criteria based on populations of interest (inclusion/ exclusion criteria), interventions (drug dosage/frequency), and outcomes (fracture assessment). The population of interest included women with PMO who were eligible to receive pharmacotherapy for primary or secondary prevention of fractures. Nine comparators were selected: eight (alendronate, denosumab, ibandronate, raloxifene, risedronate, strontium ranelate, teriparatide, and zoledronic acid) on the basis of National Institute for Health and Care Excellence recommendations of available osteoporosis treatments plus the investigational treatment romosozumab. Search terms were specific to disease, type of study, drugs, combined free text, and Medical Subject Headings (MeSH). We restricted the search to Englishlanguage publications. Exclusion criteria for the literature review included non-RCTs, phase 1 trials, letters, editorials, case reports, comments, studies not involving humans, and trials reporting only bone mineral density (BMD), a surrogate for treatment response.

\section{Feasibility assessment}

Group data for the NMA were obtained for four types of fracture: VF, NVF, wrist, and hip. Studies that did not provide sufficient information to allow derivation of RR or that reported zero events were excluded. Determination of the RR of fracture was contingent on studies reporting the number of patients in each study arm $(N)$ and the number $(n)$ or percentage of patients with fractures in each study arm.

A secondary article review was conducted to identify studies that reported on the RR of fragility fractures and that would therefore qualify for the indirect treatment comparisons. With the exception of romosozumab studies, only trials examining licensed dosages of a single agent for postmenopausal osteoporosis were included, as defined by the European Medicines Agency. Studies that included both a licensed and a nonlicensed dosage were considered only if it was possible to separate fracture outcomes in study arms by dosage. Dose-ranging studies without a control arm and switching studies assessing only a sequence of treatments were excluded, as were studies comparing the same active drug but only assessing the addition of a 
supplement in 1 of the study arms. Major osteoporotic fractures and clinical fractures were not considered for the NMA, because their definitions varied widely across studies.

After the secondary article review, an assessment was made to see if it was feasible to connect networks between treatments. The patient population characteristics of the remaining studies were then reviewed for differences in age, ethnicity, BMD at baseline, previous fractures, fracture definition and assessment, outcome measures (efficacy or safety outcomes), and previous treatments to assure the exchangeability of patient data across trials. Studies that exhibited heterogeneity factors in these patient population criteria (Supplementary Fig. 2) that would prevent adequate exchangeability of patient data across trials were excluded from the base case analysis.

\section{Meta-analyses}

We ran pair-wise meta-analyses (performed in Stata 14.1) when data comparing the same two treatments for the same outcome were available. Results from these comparisons were used primarily to check for statistical heterogeneity and for inconsistencies in the results obtained from the indirect treatment comparisons. Results were pooled by means of fixedeffects models that used the Cochran-Mantel-Haenszel method [10] and random-effects models that used the DerSimonian and Laird model [11]. The estimate of heterogeneity was taken from the Cochran-Mantel-Haenszel method and was assessed by both Cochran's $Q$ test and $I^{2}$ statistics.

Differences in trial duration were taken into account, because the time periods during which individuals experienced at least one fragility fracture varied significantly across studies. An underlying Poisson process was, therefore, assumed for each trial arm, so the time until a fracture occurred followed an exponential distribution. Network analyses were implemented using both fixed-effect and random-effects approaches. The models were fitted to the data using Bayesian Markov Chain Monte Carlo methods (Gibbs sampling) and were implemented in WinBUGS 1.4.3 (University of Cambridge MRC Biostatistics Unit, Cambridge, England, UK).

Fig. 1 Relative risk of treatments versus placebo in the vertebral fractures network. Treatment effects were significantly different for all treatments versus placebo

\section{Model selection}

As the validity of the results relied on the model converging in a satisfactory manner, we made a visual assessment at the end of each simulation using history trace plots, Brook-GelmanRubin plots, smoothed kernel posterior density plots, and autocorrelation plots. Both fixed-effects and random-effects approaches were run for each model, with the most appropriate model being selected on the basis of the total residual deviance and deviance information criterion. Using this information, we concluded that the fixed-effects model was a better fit for the data. The Cochran $Q$ test and $I^{2}$ statistics generated for each pair-wise comparison in each network confirmed this assumption in most cases.

\section{Sensitivity analyses}

Each of the networks was subjected to two sensitivity analyses. One analysis assessed the effect of removing strontium ranelate trials from the networks, because the drug's manufacturer ceased its distribution in 2017 [12]. A second sensitivity analysis assessed the impact of studies with low or ambiguous quality of evidence (e.g., studies reporting fracture outcomes as adverse events) on the NMA findings by excluding the Alendronate Phase III Osteoporosis Treatment Study Group (APOTSG) [13], EVista Alendronate Comparison (EVA) [14], and Bone Mineral Density-MultiNational (BMD-MN) trials [15].

\section{Results}

\section{Study characteristics}

After removing studies duplicated in the three databases $(n=$ 3054), we screened 4978 articles on the basis of title and abstract (Supplementary Fig. 1). An additional 4252 articles were excluded for failing to meet inclusion criteria, leaving 726 articles for full-text screening. Twenty-nine of these could not be found. Of the remaining 697 publications that

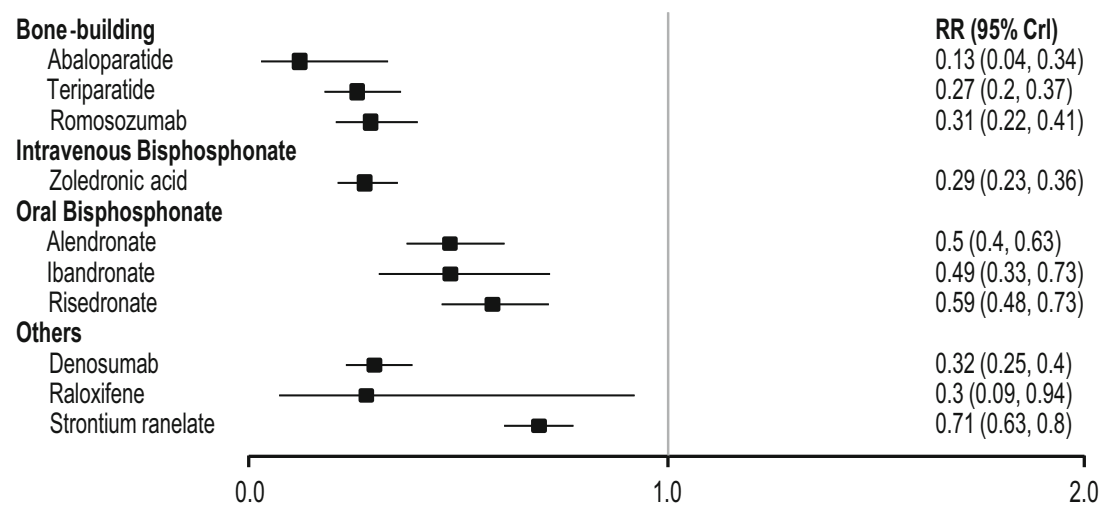




\begin{tabular}{|c|c|c|c|c|c|c|c|c|c|c|c|}
\hline Ranking & $\begin{array}{c}1 \\
\text { Most } \\
\text { effective }\end{array}$ & 2 & 3 & 4 & 5 & 6 & 7 & 8 & 9 & 10 & $\begin{array}{c}11 \\
\text { Least } \\
\text { effective }\end{array}$ \\
\hline \multicolumn{12}{|l|}{ Treatment } \\
\hline Abaloparatide & $79 \%$ & $13 \%$ & $3 \%$ & $2 \%$ & $2 \%$ & $1 \%$ & $0 \%$ & $0 \%$ & $0 \%$ & $0 \%$ & $0 \%$ \\
\hline eriparatide & $4 \%$ & $29 \%$ & $28 \%$ & $18 \%$ & $13 \%$ & $7 \%$ & $0 \%$ & $0 \%$ & $0 \%$ & $0 \%$ & $0 \%$ \\
\hline Zoledronic acid & $2 \%$ & $16 \%$ & $28 \%$ & $27 \%$ & $19 \%$ & $8 \%$ & $0 \%$ & $0 \%$ & $0 \%$ & $0 \%$ & $0 \%$ \\
\hline Romosozumab & $1 \%$ & $10 \%$ & $19 \%$ & $23 \%$ & $27 \%$ & $18 \%$ & $1 \%$ & $0 \%$ & $0 \%$ & $0 \%$ & $0 \%$ \\
\hline Denosumab & $1 \%$ & $6 \%$ & $14 \%$ & $23 \%$ & $31 \%$ & $24 \%$ & $2 \%$ & $0 \%$ & $0 \%$ & $0 \%$ & $0 \%$ \\
\hline Ibandronate & $0 \%$ & $0 \%$ & $0 \%$ & $1 \%$ & $2 \%$ & $15 \%$ & $36 \%$ & $28 \%$ & $14 \%$ & $4 \%$ & $0 \%$ \\
\hline Raloxifene & $14 \%$ & $26 \%$ & $7 \%$ & $6 \%$ & $7 \%$ & $16 \%$ & $7 \%$ & $5 \%$ & $4 \%$ & $5 \%$ & $2 \%$ \\
\hline Alendronate & $0 \%$ & $0 \%$ & $0 \%$ & $0 \%$ & $0 \%$ & $8 \%$ & $44 \%$ & $38 \%$ & $8 \%$ & $0 \%$ & $0 \%$ \\
\hline Risedronate & $0 \%$ & $0 \%$ & $0 \%$ & $0 \%$ & $0 \%$ & $1 \%$ & $8 \%$ & $27 \%$ & $57 \%$ & $7 \%$ & $0 \%$ \\
\hline Strontium ranelate & $0 \%$ & $0 \%$ & $0 \%$ & $0 \%$ & $0 \%$ & $0 \%$ & $0 \%$ & $1 \%$ & $16 \%$ & $82 \%$ & $0 \%$ \\
\hline Placebo & $0 \%$ & $0 \%$ & $0 \%$ & $\%$ & $0 \%$ & $0 \%$ & $0 \%$ & $0 \%$ & $0 \%$ & $2 \%$ & $98 \%$ \\
\hline
\end{tabular}

b

Ranking $\begin{array}{ccccccccccc}1 & 2 & 3 & 4 & 5 & 6 & 7 & 8 & 9 & 10 & \begin{array}{c}11 \\ \text { Most } \\ \text { effective }\end{array}\end{array}$

\begin{tabular}{|c|c|c|c|c|c|c|c|c|c|c|c|}
\hline Treatment & & & & & & & & & & & \\
\hline Abaloparatide & $70 \%$ & $14 \%$ & $7 \%$ & $3 \%$ & $2 \%$ & $1 \%$ & $1 \%$ & $1 \%$ & $1 \%$ & $0 \%$ & $0 \%$ \\
\hline Teriparatide & $15 \%$ & $44 \%$ & $25 \%$ & $8 \%$ & $4 \%$ & $2 \%$ & $1 \%$ & $1 \%$ & $0 \%$ & $0 \%$ & $0 \%$ \\
\hline Romosozumab & $14 \%$ & $34 \%$ & $34 \%$ & $9 \%$ & $4 \%$ & $2 \%$ & $1 \%$ & $1 \%$ & $0 \%$ & $0 \%$ & $0 \%$ \\
\hline Alendronate & $0 \%$ & $2 \%$ & $8 \%$ & $23 \%$ & $24 \%$ & $18 \%$ & $13 \%$ & $8 \%$ & $3 \%$ & $0 \%$ & $0 \%$ \\
\hline Zoledronic acid & $1 \%$ & $5 \%$ & $17 \%$ & $32 \%$ & $22 \%$ & $13 \%$ & $7 \%$ & $3 \%$ & $1 \%$ & $0 \%$ & $0 \%$ \\
\hline Strontium ranelate & $0 \%$ & $0 \%$ & $0 \%$ & $1 \%$ & $4 \%$ & $9 \%$ & $19 \%$ & $35 \%$ & $27 \%$ & $4 \%$ & $0 \%$ \\
\hline Risedronate & $0 \%$ & $0 \%$ & $2 \%$ & $8 \%$ & $18 \%$ & $26 \%$ & $25 \%$ & $15 \%$ & $5 \%$ & $0 \%$ & $0 \%$ \\
\hline Denosumab & $0 \%$ & $1 \%$ & $4 \%$ & $11 \%$ & $16 \%$ & $20 \%$ & $21 \%$ & $17 \%$ & $9 \%$ & $1 \%$ & $0 \%$ \\
\hline Raloxifene & $0 \%$ & $1 \%$ & $2 \%$ & $4 \%$ & $6 \%$ & $7 \%$ & $10 \%$ & $16 \%$ & $28 \%$ & $17 \%$ & $10 \%$ \\
\hline Placebo & $0 \%$ & $0 \%$ & $0 \%$ & $0 \%$ & $0 \%$ & $0 \%$ & $0 \%$ & $1 \%$ & $19 \%$ & $63 \%$ & $18 \%$ \\
\hline Ibandronate & $0 \%$ & $0 \%$ & $0 \%$ & $0 \%$ & $0 \%$ & $1 \%$ & $1 \%$ & $3 \%$ & $8 \%$ & $14 \%$ & $72 \%$ \\
\hline
\end{tabular}

$\begin{array}{cccccccccc}\text { C } & 1 & 2 & 3 & 4 & 5 & 6 & 7 & 8 \\ \begin{array}{c}\text { Most } \\ \text { effective }\end{array} & & & & & & & \begin{array}{c}\text { Least } \\ \text { effective }\end{array}\end{array}$

Treatment

effective

\begin{tabular}{|c|c|c|c|c|c|c|c|c|}
\hline Abaloparatide & $53 \%$ & $21 \%$ & $15 \%$ & $6 \%$ & $2 \%$ & $1 \%$ & $1 \%$ & $0 \%$ \\
\hline Alendronate & $26 \%$ & $47 \%$ & $22 \%$ & $4 \%$ & $1 \%$ & $0 \%$ & $0 \%$ & $0 \%$ \\
\hline Risedronate & $3 \%$ & $13 \%$ & $29 \%$ & $32 \%$ & $13 \%$ & $6 \%$ & $3 \%$ & $2 \%$ \\
\hline Teriparatide & $0 \%$ & $2 \%$ & $8 \%$ & $17 \%$ & $21 \%$ & $16 \%$ & $13 \%$ & $22 \%$ \\
\hline Raloxifene & $17 \%$ & $17 \%$ & $18 \%$ & $13 \%$ & $8 \%$ & $5 \%$ & $5 \%$ & $18 \%$ \\
\hline Denosumab & $0 \%$ & $1 \%$ & $6 \%$ & $21 \%$ & $34 \%$ & $24 \%$ & $10 \%$ & $5 \%$ \\
\hline Placebo & $0 \%$ & $0 \%$ & $0 \%$ & $1 \%$ & $6 \%$ & $25 \%$ & $43 \%$ & $25 \%$ \\
\hline Strontium ranelate & $0 \%$ & $0 \%$ & $1 \%$ & $6 \%$ & $15 \%$ & $23 \%$ & $26 \%$ & $28 \%$ \\
\hline
\end{tabular}


Fig. 2 Treatment ranking of osteoporosis treatments in the network metaanalysis. 11 osteoporosis treatments in the vertebral fracture network (fixed-effects model) (a). 11 osteoporosis treatments in the nonvertebral fracture network (fixed-effects model) (b). 8 osteoporosis treatments in the wrist fracture network (fixed-effects model) (c) Circles denote highest probabilities for each treatment

underwent full-text review, 76 articles were included for data extraction.

Following secondary article review, 56 distinct studies emerged for use in the indirect treatment comparisons. Of these, 25 studies reported on VF, 25 on NVF, 18 on hip fracture, 11 on wrist fracture, 17 on clinical fractures, and 4 on major osteoporotic fractures and were deemed suitable for the networks (Supplementary Fig. 2). Thirty-four studies qualified for data extraction; however, 12 were excluded because their patient population characteristics were considered to be too different from those of patients in ACTIVE or because they focused on clinical or major osteoporotic fractures. Thus, a total of 22 studies remained for inclusion in the 3 fracture networks (VF, NVF, and wrist; Supplementary Table 1). Baseline characteristics of studies included in the networks and reasons for excluding studies from the networks due to heterogeneity factors are shown in Supplementary Tables 2 and 3 .

The time periods during which individuals experienced at least one fragility fracture ranged from 12 to 60 months postbaseline across studies in the NMA.

\section{Vertebral fractures network}

Of the 25 RCTs providing suitable VF data, 7 studies were excluded because their patient population characteristics were considered to be too different from those of patients in ACTIVE (Supplementary Table 1). The final analysis therefore comprised $18 \mathrm{VF}$ studies comparing 11 treatments in 40,901 women with PMO (Supplementary Fig. 3).

Figure 1 presents relative risk data with placebo as the reference treatment. All treatments exhibited superior efficacy

Fig. 3 Relative risk of treatments versus placebo in the nonvertebral fracture network. ${ }^{\mathrm{a}}$ Abaloparatide effect significantly different from network treatment to placebo, and all treatment effects were statistically significant versus placebo for preventing VF $(p<0.05)$. Abaloparatide had the greatest effect versus placebo $(\mathrm{RR}=$ $0.13 ; 95 \%$ credible interval [CrI] $0.04-0.34)$, followed by teriparatide $(\mathrm{RR}=0.27 ; 95 \% \mathrm{CrI} 0.20-0.37)$ and zoledronic acid $(\mathrm{RR}=0.29 ; 95 \% \mathrm{CrI} 0.23-0.36)$. Using abaloparatide as reference treatment, abaloparatide was significantly more effective compared with strontium ranelate and all oral bisphosphonates; however, no significant differences emerged versus teriparatide, denosumab, raloxifene, zoledronic acid, or romosozumab (Supplementary Fig. 4). Abaloparatide was ranked first among all treatments, with a $79 \%$ estimated probability of being the most effective agent for preventing VF (Fig. 2a). The second highest estimated probability, 29\%, was accorded to teriparatide.

\section{Nonvertebral fractures network}

Four of the 25 RCTs providing suitable NVF data were excluded because their patient population characteristics were considered to be too different from those of patients in ACTIVE (Supplementary Table 1). Thus, 21 studies remained, comparing 11 treatments in 62,606 women with PMO for inclusion in the final NVF analysis (Supplementary Table 1, Supplementary Fig. 5).

Figure 3 shows relative risk data with placebo as the reference treatment. All treatments except ibandronate had a beneficial treatment effect in preventing NVF relative to placebo, although the effect for raloxifene was not statistically significant at the $p<0.05$ level. Abaloparatide had the greatest treatment effect $(\mathrm{RR}=0.50 ; 95 \%$ CrI $0.28-0.85)$, followed by teriparatide $(\mathrm{RR}=0.62 ; 95 \% \mathrm{CrI} 0.47-0.82)$ and romosozumab ( $\mathrm{RR}=0.64 ; 95 \%$ CrI 0.49-0.81). Abaloparatide was also significantly more effective compared with ibandronate and strontium ranelate (Supplementary Fig. 6). Abaloparatide was ranked first among osteoporosis treatments, with a $70 \%$ estimated probability of being the most effective agent for preventing NVF (Fig. 2b); teriparatide was ranked second with a $44 \%$ probability.

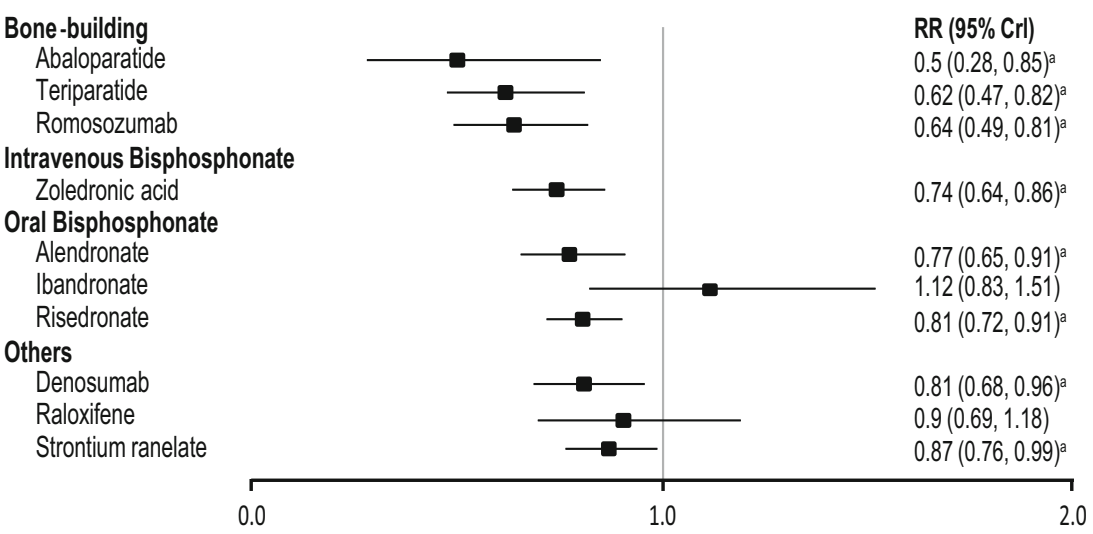




\section{Wrist fractures}

Of the 11 RCTs providing suitable wrist data, 1 was excluded because its patient population characteristics were considered to be too different from those of patients in ACTIVE (Supplementary Table 1). Ten RCTs comparing 8 treatments in 24,523 women with PMO were included in the wrist fractures network (Supplementary Table 1, Supplementary Fig. 7). Figure 4 presents relative risk data with placebo as the reference treatment. Beneficial effects in preventing wrist fracture relative to placebo were statistically significant for abaloparatide and alendronate only $(p<0.05)$. Abaloparatide had the greatest treatment effect $(\mathrm{RR}=0.39$; $95 \% \mathrm{CrI} 0.15-$ $0.90)$, followed by alendronate $(\mathrm{RR}=0.46$; $\mathrm{CrI} 0.29-0.70)$ and raloxifene $(\mathrm{RR}=0.63$; $\mathrm{CrI} 0.20-2.09)$. Abaloparatide was also significantly more effective at preventing wrist fracture than strontium ranelate (Supplementary Fig. 8). Abaloparatide was ranked as having a 53\% estimated probability of being the most effective treatment to prevent wrist fracture (Fig. 2c); alendronate was ranked second with a $47 \%$ probability.

\section{Sensitivity analyses}

Excluding strontium ranelate from the main analysis occasioned very minor changes in the results (Supplementary Tables 4, 5, and 6). Similarly, exclusion of studies providing low or unclear quality of evidence had a minimal impact on NMA findings.

\section{Hip fractures}

In ACTIVE, there were two hip fractures in the placebo group and zero in the abaloparatide group [7]. As ACTIVE was the only abaloparatide study included in the NMA, the absence of events in the abaloparatide group caused convergence issues in the hip network. Attempts to compensate for the lack of hip event data using methods from pairwise frequentist metaanalyses resulted in estimated treatment effects that lacked sufficient precision for inclusion in this study.

\section{Discussion}

Meta-analyses, although a critical tool for analyzing results of multiple independent studies, can only make pair-wise comparisons of treatments. NMAs, by contrast, synthesize information over a network of comparisons to assess the relative effects of multiple interventions used for the same condition. NMA employs both direct and indirect evidence in a general statistical framework to generate estimates that integrate all available data [16]. In bypassing the limitations of traditional pair-wise meta-analyses, NMAs enable researchers to rank the relative efficacy of all interventions, including those that have not been compared directly in head-to-head trials. NMAs therefore provide crucial information to clinicians.

Our NMA was undertaken to provide evidence regarding the relative efficacy of 10 treatments for postmenopausal women with osteoporosis who are at high risk of fragility fractures. The final analysis comprised 22 RCTs yielding usable data for 3 types of fractures: 18 RCTs with data on VF, 21 with data on NVF, and 10 with data on wrist fracture. The individual networks revealed that abaloparatide offered postmenopausal women with and without prior fractures the greatest treatment effect relative to placebo, compared with other available treatment options for all fracture types under consideration. Furthermore, treatment ranking indicated that abaloparatide had the highest estimated probability of preventing fractures in each network: $79 \%$ for $\mathrm{VF}, 70 \%$ for NVF, and $53 \%$ for wrist fracture. Of note, each network demonstrated a good level of agreement with the direct trial evidence and direct pair-wise comparisons.

The validity of NMAs depends on the comparability of patients across trials. This means that all included studies are measuring the same relative treatment effects, and any observed differences are due to chance. Put differently, all treatments could have been included in the same study and, therefore, could be viewed as truly competing interventions [17]. By this standard, we believe that our NMA is valid. The strength of our NMA rests on several factors, key among them being the use of a systematic and comprehensive approach to capturing data. Systematic reviews of RCTs are germane to the development of evidence-based medicine and yield highquality information when performed in a rigorous manner.
Fig. 4 Relative risk of treatments versus placebo in the wrist fracture network. ${ }^{\mathrm{a}}$ Abaloparatide effect significantly different from network treatment
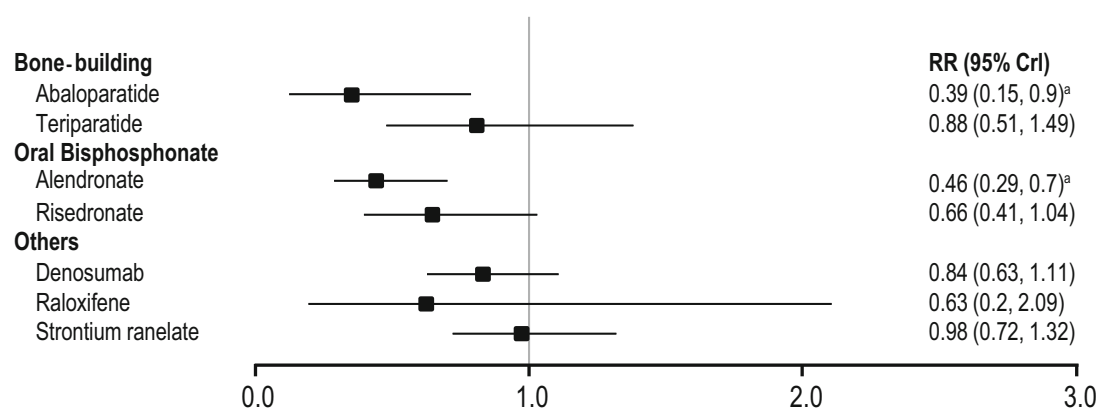
The robustness of our RCT data was confirmed by the Cochrane Risk of Bias Tool and dual reviews of bias assessments. Additionally, the RCTs underwent both a feasibility assessment and a secondary review to validate their fitness for indirect treatment comparisons. The heterogeneity analysis indicated low or no heterogeneity between studies except when studies on strontium ranelate were included in comparisons of the vertebral and nonvertebral fractures networks. In these cases, the high or moderate heterogeneity may have been due to clinical or methodologic differences between the TReatment Of Peripheral OSteoporosis (TROPOS) [18] and Spinal Osteoporosis Therapeutic Intervention (SOTI) trials [19], as patients in the TROPOS trial were older than patients in the SOTI trial (mean age 76.7 years and 69.4 years, respectively) and had a different history of VF at baseline. To further ensure the quality of the data, we ran two sensitivity analyses: The first one assessed the effect of removing the strontium ranelate studies because of the withdrawal of the agent; the other assessed the impact of studies with low or ambiguous quality of evidence. Both analyses demonstrated a minimal impact on NMA findings. We selected Bayesian Markov Chain Monte Carlo methods to fit the models to the data, because a Bayesian NMA provides a flexible framework by which to allow for complexity in the data [16].

To our knowledge, there have been five NMAs of osteoporosis treatments previously published in peer-reviewed journals, none of which included abaloparatide [20-24]. Recently, an NMA including abaloparatide was prepared for the California Technology Assessment Forum (CTAF), a core program of the Institute for Clinical and Economic Review (ICER) that publicly evaluates objective evidence reports and recommends how evidence can be used to improve the quality and value of health care [25]. This NMA was published in an ICER evidence report and included two anabolic agents, abaloparatide and teriparatide, as well as zoledronic acid [25]. The RCTs used in the networks enrolled postmenopausal women at high risk for a fragility fracture, and all were placebo-controlled [25]. Findings largely corroborate those from our research. In the VF network, all active treatments performed significantly better than placebo, with the RR of VF fracture being 0.13 (CrI 0.03-0.33) for abaloparatide, 0.17 (CrI 0.09-0.29) for teriparatide, and 0.30 (CrI 0.24-0.37) for zoledronic acid [25]. In the NVF network, each of the active therapies significantly reduced fracture risk compared with placebo, with the RR of NVF being 0.51 (CrI 0.28-0.85) for abaloparatide, 0.61 (CrI 0.41-0.88) for teriparatide, and 0.75 (CrI 0.64-0.87) for zoledronic acid [25]. Wrist fracture data were not reported, and the benefits of treatments for hip fracture were judged to be uncertain. The CTAF report concluded that, when active agents were compared with placebo, there was (1) moderate certainty that anabolic agents provided a small or substantial net health benefit and (2) high certainty that they provided at least a small net health benefit. These conclusions were based on the NMA showing a substantial reduction in VF and a small-to-moderate reduction in NVF [25].

Our systematic review and NMA have several limitations. First, we confined our data searches to English-only publications, which meant that 35 non-English publications were excluded; however, that particular restriction has not been shown previously to bias systematic reviews and meta-analyses [26]. Though several studies were excluded based on heterogeneity factors as described above, differences in the designs and population characteristics of the studies included in the NMA represent a second limitation. Additionally, comparators evaluated in our NMA were restricted to those included in National Institute for Health and Care Excellence recommendations of available osteoporosis treatments plus the investigational treatment romosozumab. Consequently, agents such as bazedoxifene, with regulatory approval in a small number of countries, are not taken into account. A possible second limitation of our NMA is that we did not consider adverse events or drug costs. It is well-known that adverse events affect adherence to bisphosphonate therapy, with one large observational study reporting only $45 \%$ compliance 1 year after initiation and only $30 \%$ compliance at 2 years [27]. Adherence to anabolic agents appears to be better, despite the requirement for daily subcutaneous injection. A small $(N=111)$ retrospective chart review showed the persistence rate with teriparatide to be $90 \%$ at 6 months and $75 \%$ at 18 months [28]. Only $20 \%$ of patients in that study cited adverse events as the reason for nonadherence.

Healthcare economics are especially relevant in the evaluation of hip fractures, which are associated with significant short-term morbidity, long-term loss of independence, nursing home placement, and increased mortality [29]. Hip fractures skew the cost distribution of fragility fractures by accounting for $72 \%$ of total outlays but only $14 \%$ of all fractures [3]. The absence of hip fracture data suggests a third limitation of our NMA, but the absence of events in the abaloparatide arm of ACTIVE [7] precluded a meaningful assessment of treatment effects on hip fractures using NMA. However, evidence from the abaloparatide extension trial points to a possible protective effect of abaloparatide treatment. ACTIVExtend enrolled 558 women from the abaloparatide group and 581 from the placebo group, all of whom were switched to alendronate, $70 \mathrm{mg}$ weekly for 2 years [8]. The cumulative incidence of hip fracture after 24 months was 5 in the group switched from placebo and zero in the group switched from abaloparatide, implying long-term risk reduction with active treatment. Additionally, a recent observational study of patients taking teriparatide $(N=$ $14,284)$ reported a statistically significant decrease in hip fracture among patients who persisted longer with treatment or had higher adherence [30]. Regardless of whether these findings represent a class effect of anabolic agents, the two reports taken together are, at the least, encouraging. 


\section{Conclusions}

Given the high level of morbidity and healthcare costs associated with fragility fractures, it is imperative to identify therapeutic agents and other treatment modalities that can reduce fracture risk. In this NMA of 10 pharmacotherapeutic agents used to treat osteoporosis, abaloparatide reduced the relative risk of VF, NVF, and wrist fracture versus placebo in postmenopausal women with or without prior fracture, compared with other treatment options. Generalizability of the findings is limited to the trial populations included in our NMA. Additionally, clinicians need to be somewhat skeptical of the conclusions of analyses that involve only indirect comparisons rather than head-to-head comparisons.

It should be noted that although anabolic agents may have a significant role in preventing fragility fractures, regulatory authorities limit the use of anabolic drugs for postmenopausal osteoporosis to 18 to 24 months [31, 32]. Furthermore, at least one study has shown that BMD gains from anabolic agents are quickly lost in the absence of follow-up therapy [33]. However, emerging evidence suggests that optimal sequencing of therapies is the key to preserving gains made on anabolic agents [34-37]. Consistent with this hypothesis, ACTIVExtend showed cumulative benefit from treatment with abaloparatide followed by an antiresorptive agent [8]. Additional fracture endpoint studies are awaited to help guide selection of the most appropriate agents and ideal duration of follow-up treatment.

Acknowledgements Editorial assistance was provided by Adelphi Communications, New York, NY.

Funding This study was supported by Radius Health, Inc., Waltham, MA.

\section{Compliance with ethical standards}

Conflicts of interest Jean-Yves Reginster has provided consultancy to, been an advisory board speaker for, or had grant support from Laboratoires Genévrier, Radius Health, Inc., Laboratoires Servier, Pierre Fabre, Cerin GmbH, Institut Biochimique SA, and Mylan N.V.

Florence Bianic, Rosanne Campbell, and Monique Martin are employees of Syneos Health, which was contracted by Radius Health, Inc. to perform this analysis.

Setareh A. Williams and Lorraine A. Fitzpatrick are employees of and shareholders in Radius Health, Inc.

Open Access This article is distributed under the terms of the Creative Commons Attribution-NonCommercial 4.0 International License (http:// creativecommons.org/licenses/by-nc/4.0/), which permits any noncommercial use, distribution, and reproduction in any medium, provided you give appropriate credit to the original author(s) and the source, provide a link to the Creative Commons license, and indicate if changes were made.

\section{References}

1. Johnell O, Kanis JA (2006) An estimate of the worldwide prevalence and disability associated with osteoporotic fractures. Osteoporos Int 17:1726-1733

2. Office of the Surgeon General (2004) Bone health and osteoporosis: a report of the surgeon general. Available at: https://www.ncbi.nlm. nih.gov/books/NBK45513/. Accessed November 7, 2018

3. Burge R, Dawson-Hughes B, Solomon DH, Wong JB, King A, Tosteson A (2007) Incidence and economic burden of osteoporosis-related fractures in the United States, 2005-2025. J Bone Miner Res 22:465-475

4. Kanis JA, McCloskey EV, Johansson H, Cooper C, Rizzoli R, Reginster J-Y (2013) European guidance for the diagnosis and management of osteoporosis in postmenopausal women. Osteoporos Int 24:23-57

5. Hattersley G, Dean T, Corbin BA, Bahar H, Gardella TJ (2016) Binding selectivity of abaloparatide for pth-type-1-receptor conformations and effects on downstream signaling. Endocrinology 157: 141-149

6. TYMLOS (abaloparatide) (2017). Radius Health, Inc., Waltham, MA. Available at: https://www.accessdata.fda.gov/drugsatfda docs/label/2017/2087431bl.pdf. Accessed November 7, 2018

7. Miller PD, Hattersley G, Riis BJ, Williams GC, Lau E, Russo LA, Alexandersen P, Zerbini CA, Hu MY, Harris AG, Fitzpatrick LA, Cosman F, Christiansen C, Study Investigators ACTIVE (2016) Effect of abaloparatide vs placebo on new vertebral fractures in postmenopausal women with osteoporosis: a randomized clinical trial. JAMA 316:722-733

8. Bone HG, Cosman F, Miller PD, Williams GC, Hattersley G, Hu MY, Fitzpatrick LA, Mitlak B, Papapoulos S, Rizzoli R, Dore RK, Bilezikian JP, Saag KG (2018) ACTIVExtend: 24 months of alendronate after 18 months of abaloparatide or placebo for postmenopausal osteoporosis. J Clin Endocrinol Metab 103:2949-2957

9. Committee for Medicinal Products for Human Use (CHMP) (2007) Guideline on the evaluation of medicinal products in the treatment of primary osteoporosis. Available at: https://www.ema.europa.eu/ documents/scientific-guideline/guideline-evaluation-medicinalproducts-treatment-primary-osteoporosis_en.pdf. Accessed November 7, 2018

10. Mantel N, Haenszel W (1959) Statistical aspects of the analysis of data from retrospective studies of disease. J Natl Cancer Inst 22: 719-748

11. DerSimonian R, Laird N (1986) Meta-analysis in clinical trials. Control Clin Trials 7:177-188

12. Servier UK (2017) Servier to cease worldwide production and supply of protelos. Available at: https:/www.servier.co.uk/sites/ default/files/media/servier protelos medical industry pr final. pdf. Accessed November 7, 2018

13. Liberman UA, Weiss SR, Broll J, Minne HW, Quan H, Bell NH, Rodriguez-Portales J, Downs RW Jr, Dequeker J, Favus M (1995) Effect of oral alendronate on bone mineral density and the incidence of fractures in postmenopausal osteoporosis. The alendronate phase III osteoporosis treatment study group. N Engl J Med 333:14371443

14. Recker RR, Kendler D, Recknor CP, Rooney TW, Lewiecki EM, Utian WH, Cauley JA, Lorraine J, Qu Y, Kulkarni PM, Gaich CL, Wong M, Plouffe L Jr, Stock JL (2007) Comparative effects of raloxifene and alendronate on fracture outcomes in postmenopausal women with low bone mass. Bone 40:843-851

15. Fogelman I, Ribot C, Smith R, Ethgen D, Sod E, Reginster JY (2000) Risedronate reverses bone loss in postmenopausal women with low bone mass: results from a multinational, double-blind, placebo-controlled trial. Bmd-mn study group. J Clin Endocrinol Metab 85:1895-1900 
16. Tu YK (2014) Use of generalized linear mixed models for network meta-analysis. Med Decis Mak 34:911-918

17. Cameron C, Fireman B, Hutton B, Clifford T, Coyle D, Wells G, Dormuth CR, Platt R, Toh S (2015) Network meta-analysis incorporating randomized controlled trials and non-randomized comparative cohort studies for assessing the safety and effectiveness of medical treatments: challenges and opportunities. Syst Rev 4:147

18. Reginster JY, Seeman E, De Vernejoul MC, Adami S, Compston J, Phenekos C, Devogelaer JP, Curiel MD, Sawicki A, Goemaere S, Sorensen OH, Felsenberg D, Meunier PJ (2005) Strontium ranelate reduces the risk of nonvertebral fractures in postmenopausal women with osteoporosis: treatment of peripheral osteoporosis (TROPOS) study. J Clin Endocrinol Metab 90:2816-2822

19. Meunier PJ, Roux C, Seeman E, Ortolani S, Badurski JE, Spector TD, Cannata J, Balogh A, Lemmel EM, Pors-Nielsen S, Rizzoli R, Genant HK, Reginster JY (2004) The effects of strontium ranelate on the risk of vertebral fracture in women with postmenopausal osteoporosis. N Engl J Med 350:459-468

20. Davis SR, Tan A, Bell RJ (2015) Targeted assessment of fracture risk in women at midlife. Osteoporos Int 26:1705-1712

21. Freemantle N, Cooper C, Diez-Perez A, Gitlin M, Radcliffe H, Shepherd S, Roux C (2013) Results of indirect and mixed treatment comparison of fracture efficacy for osteoporosis treatments: a metaanalysis. Osteoporos Int 24:209-217

22. Murad MH, Drake MT, Mullan RJ, Mauck KF, Stuart LM, Lane MA, Abu Elnour NO, Erwin PJ, Hazem A, Puhan MA, Li T, Montori VM (2012) Clinical review. Comparative effectiveness of drug treatments to prevent fragility fractures: a systematic review and network meta-analysis. J Clin Endocrinol Metab 97:1871-1880

23. Yang XC, Deng ZH, Wen T, Luo W, Xiao WF, Zhao RB, Li YS (2016) Network meta-analysis of pharmacological agents for osteoporosis treatment and fracture prevention. Cell Physiol Biochem 40:781-795

24. Zhang L, Pang Y, Shi Y, Xu M, Xu X, Zhang J, Ji L, Zhao D (2015) Indirect comparison of teriparatide, denosumab, and oral bisphosphonates for the prevention of vertebral and nonvertebral fractures in postmenopausal women with osteoporosis. Menopause 22:1021-102550

25. Institute for Clinical and Economic Review (2017) Anabolic therapies for osteoporosis in postmenopausal women: effectiveness an value. Available at: https://icer-review.org/wp-content/uploads/ 2016/11/CTAF Osteoporosis Evidence Report 061617.pdf. Accessed November 7, 2018

26. Morrison A, Polisena J, Husereau D, Moulton K, Clark M, Fiander M, Mierzwinski-Urban M, Clifford T, Hutton B, Rabb D (2012) The effect of English-language restriction on systematic reviewbased meta-analyses: a systematic review of empirical studies. Int J Technol Assess Health Care 28:138-144
27. Karlsson L, Lundkvist J, Psachoulia E, Intorcia M, Strom O (2015) Persistence with denosumab and persistence with oral bisphosphonates for the treatment of postmenopausal osteoporosis: a retrospective, observational study, and a meta-analysis. Osteoporos Int 26:2401-2411

28. Mulgund M, Beattie KA, Wong AK, Papaioannou A, Adachi JD (2009) Assessing adherence to teriparatide therapy, causes of nonadherence and effect of adherence on bone mineral density measurements in osteoporotic patients at high risk for fracture. Ther Adv Musculoskelet Dis 1:5-11

29. Braithwaite RS, Col NF, Wong JB (2003) Estimating hip fracture morbidity, mortality and costs. J Am Geriatr Soc 51:364-370

30. Burge RT, Disch DP, Gelwicks S, Zhang X, Krege JH (2017) Hip and other fragility fracture incidence in real-world teriparatide-treated patients in the United States. Osteoporos Int 28:799-809

31. Forteo (teriparatide) (2012) Eli Lilly and Co., Indianapolis, IN. Available at: https://www.accessdata.fda.gov/drugsatfda docs/ label/2009/021318s012lbl.pdf. Accessed November 7, 2018

32. Forteo Teriparatide (rbe) Injection (2015) Eli Lilly Australia Pty Limited, West Ryde NSW. Available at: https://www.ebs.tga.gov. $\mathrm{au} / \mathrm{ebs} / \mathrm{picmi} / \mathrm{picmirepository} \cdot \mathrm{nsf} / \mathrm{PICMI}$ ?OpenForm\&t=\&q= teriparatide. Accessed November 7, 2018

33. Black DM, Bilezikian JP, Ensrud KE, Greenspan SL, Palermo L, Hue T, Lang TF, McGowan JA, Rosen CJ (2005) One year of alendronate after one year of parathyroid hormone (1-84) for osteoporosis. N Engl J Med 353:555-565

34. Leder BZ, Tsai JN, Jiang LA, Lee H (2017) Importance of prompt antiresorptive therapy in postmenopausal women discontinuing teriparatide or denosumab: the denosumab and teriparatide follow-up study (data-follow-up). Bone 98:54-58

35. Leder BZ, Tsai JN, Uihlein AV, Wallace PM, Lee H, Neer RM, Burnett-Bowie SA (2015) Denosumab and teriparatide transitions in postmenopausal osteoporosis (the data-switch study): extension of a randomised controlled trial. Lancet 386:1147-1155

36. Rittmaster RS, Bolognese M, Ettinger MP, Hanley DA, Hodsman AB, Kendler DL, Rosen CJ (2000) Enhancement of bone mass in osteoporotic women with parathyroid hormone followed by alendronate. J Clin Endocrinol Metab 85:2129-2134

37. Saag KG, Petersen J, Brandi ML, Karaplis AC, Lorentzon M, Thomas T, Maddox J, Fan M, Meisner PD, Grauer A (2017) Romosozumab or alendronate for fracture prevention in women with osteoporosis. N Engl J Med 377:1417-1427 